\title{
Establishing acceptance regions for $L$-moments based goodness-of-fit tests by stochastic simulation
}

\author{
Jun-Jih Liou, Yii-Chen Wu, Ke-Sheng Cheng *
}

Department of Bioenvironmental Systems Engineering, National Taiwan University, Taipei, Taiwan, ROC

Received 29 December 2006; received in revised form 26 February 2008; accepted 28 February 2008

\section{KEYWORDS \\ L-moments; \\ Frequency analysis; \\ Goodness-of-fit test; \\ Stochastic simulation; \\ Acceptance regions}

\begin{abstract}
Summary Before conducting a hydrological frequency analysis the best-fit distribution for the hydrological variable of interest must be decided by a goodness-of-fit test or other appropriate methods. In recent years the $L$-moment-ratio diagram has been suggested as a useful tool for discrimination between candidate distributions. However, few research works have been conducted on the effect of sample size on goodness-of-fit test using the $L$-moment-ratio diagram. In this study, through stochastic simulation, statistical properties of two estimators, namely the probability-weighted-moment estimator and the plotting-position estimator, of the $L$-skewness and $L$-kurtosis of the normal and Gumbel distributions are discussed. The joint distribution of the sample $L$-skewness and $L$-kurtosis is found to be approximately bivariate normal for larger sample sizes. Consequently, a set of sample-size-dependent $95 \%$ acceptance regions for L-moments-based goodness-of-fit tests of the normal and Gumbel distributions was established using stochastic simulation technique. Such acceptance regions were further validated using simulated random samples, with regard to the consistence of the acceptance rate and the desired level of significance, and were found to be applicable for goodness-of-fit tests for random samples of any sample size between 20 and 1000 .

(c) 2008 Elsevier B.V. All rights reserved.
\end{abstract}

\section{Introduction}

Hydrological frequency analysis is one of the essential tasks in hydrological engineering design. It is the work of determining the magnitudes of hydrological variables corresponding to given frequencies or recurrence intervals.

\footnotetext{
* Corresponding author. Tel.: +88622366 1568; fax: +88622363 5854.

E-mail address: rslab@ntu.edu.tw (K.-S. Cheng).
}

Procedures involved in frequency analysis include: (1) collecting a random sample of the interested hydrological variable; (2) finding the best-fit distribution for the sample by a goodness-of-fit (GOF) test or other appropriate methods; and (3) determining the magnitude of the hydrological variable corresponding to a given probability of exceedance using the best-fit distribution. Chow (1951) proposed the following general equation for hydrological frequency analysis of a random variable $X$ with mean $\mu$ and standard deviation $\sigma$ : 
$x_{T}=\mu+K_{T} \sigma$

where $K_{T}$, the frequency factor, is a function of the return period $T$ and is dependent on the distribution type of $X$. Frequency factors of distributions commonly used in hydrological frequency analysis are themselves random variables and their distributions have been identified (Kite, 1988). Upon collection of a random sample, one must decide the type of distribution which best characterizes the random sample and determine the corresponding $K_{T}$ value.

Two GOF tests, namely the chi-square test and the Kolmogorov-Smirnov test, are often used for the selection of probability distributions for hydrological variables (Haan, 2002). Another method of goodness-of-fit test is the method based on ordinary moment-ratio diagrams (D'Agostino and Stephens, 1986). Moment ratios are unique properties of probability distributions and sample moment ratios of ordinary skewness and kurtosis have been used for selection of probability distribution (Kottegoda, 1980; D'Agostino and Stephens, 1986). In recent years there have been many applications of $L$-moments for frequency analysis and the skewness and kurtosis L-moment-ratio diagram (LMRD) was suggested as a useful tool for discrimination between candidate distributions (Hosking, 1990; Hosking and Wallis, 1993, 1997; Vogel and Fennesset, 1993). The L-moments uniquely define the distribution if the mean of the distribution exists, and the $L$-skewness and $L$-kurtosis are much less biased than the ordinary skewness and kurtosis (Hosking and Wallis, 1997).

A two-parameter distribution with a location and a scale parameter plots as a single point on the LMRD, whereas a three-parameter distribution with location, scale and shape parameters plots as a curve on the LMRD, and distributions with more than one shape parameter generally are associated with regions on the diagram (Hosking and Wallis, 1997). However, theoretical points or curves of various probability distributions on the LMRD cannot accommodate for uncertainties induced by parameter estimation using random samples. Although several graphic methods have been developed for determination of the best-fit distribution (Vogel et al., 1993; Hosking and Wallis, 1995; Vogel and Wilson, 1996; Chow and Watt, 1994), the issue of how well a distribution fits the sample $L$-moment ratios has not been fully investigated in the literature. In addition to GOF tests for at-site sets of data, $L$-moments can also be used to produce a set of likely candidate distributions for regional frequency analysis (Wallis, 1988) which generally requires a GOF test for interrelated multi-sites data. The multi-sites data usually have different record lengths and their estimates of parameters have different accuracies. Therefore, the effect of record length on estimates of parameters should be considered in determining the bestfit distribution for regional frequency analysis. Additionally, for the chi-square and the Kolmogorov-Smirnov tests, the sample size (equivalent to the record length) is a factor affecting the decision on rejection or acceptance of the null hypothesis. Therefore, it is also desirable to take the sample size into consideration when a GOF-test based on L-moments is implemented. To date, few research works focusing on the effect of sample size on GOF test using the LMRD have been conducted. Consequently, the objectives of this study are: (1) to investigate the feasibility of establishing acceptance regions for $L$-moments-based GOF test and (2) to investigate the effect of sample size on the acceptance regions using stochastic simulation. The normal and Gumbel distributions are adopted to exemplify the proposed method.

This paper is organized as follows. Section 'The ordinary moment-ratio diagram' contains a brief description of the ordinary moment ratios and the joint distribution of the sample skewness and kurtosis. Section ' $L$-moments and the $L$-moment-ratio diagram' defines the $L$-moment-ratios and their probability-weighted-moment and plotting-position estimators. Section 'Stochastic simulation of normal and Gumbel distributions' describes details of random samples generation for the normal and Gumbel distributions using stochastic simulation. Detailed discussions on the properties of the sample $L$-skewness and $L$-kurtosis are also given. Section 'Evaluating the bivariate normality assumption for sample $L$-skewness and $L$-kurtosis using the Mardia test' introduces the Mardia test for test of bivariate normality of sample $L$-skewness and $L$-kurtosis. Section 'Establishing acceptance regions for GOF tests' gives a detailed account on how the 95\% acceptance regions of GOF tests based on $L$-moment-ratios of the normal and Gumbel distributions can be established. Section 'Empirical relationships between parameters of acceptance regions and sample size' extends the $95 \%$ acceptance regions to be used for any sample size by establishing empirical relationships between parameters of the $95 \%$ acceptance regions and the sample size. Section 'Validity check of the LMRD acceptance regions' describes the procedures and results of a validity check of the sample-size-dependent 95\% acceptance regions of the $L$-moments-based GOF tests.

\section{The ordinary moment-ratio diagram}

Parameters of a probability distribution can be expressed in terms of its moments $\left(\mu_{r}^{\prime}\right)$ or central moments $\left(\mu_{r}\right)$ defined as

$\mu_{r}^{\prime}=E\left(X^{r}\right)$

$\mu_{r}=E\left(X-\mu_{1}^{\prime}\right)^{r}$

for $r=1,2, \ldots$. Therefore, the shape of a probability distribution can be characterized by the moments of that distribution. This is achieved by using the relationships between the standardized coefficient of skewness $\left(\sqrt{\beta_{1}}\right)$ and kurtosis $\left(\beta_{2}\right)$ which are defined as

$\sqrt{\beta_{1}}=\frac{\mu_{3}}{\left(\mu_{2}\right)^{3 / 2}}$
$\beta_{2}=\frac{\mu_{4}}{\left(\mu_{2}\right)^{2}}$

Theoretical $\sqrt{\beta_{1}} \sim \beta_{2}$ relationships of various distributions (Fig. 1) are known as the moment-ratio diagram. Hereafter, the standardized moments $\sqrt{\beta_{1}}$ and $\beta_{2}$ will be referred to as the skewness and kurtosis, respectively.

Given a random sample $\left\{x_{1}, x_{2}, \ldots, x_{n}\right\}$, the sample estimates of $\sqrt{\beta_{1}}$ and $\beta_{2}$ are defined as

$\sqrt{b_{1}}=\frac{m_{3}}{s^{3}}$
$b_{2}=\frac{m_{4}}{s^{4}}$ 


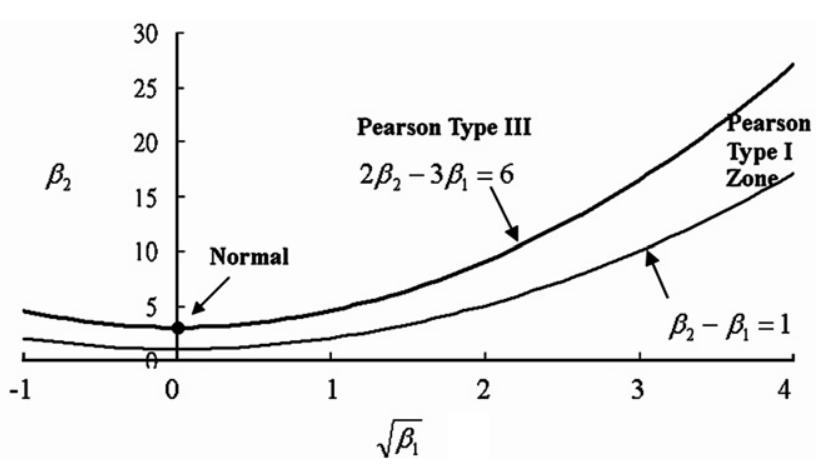

Figure 1 Ordinary moment-ratio diagram.

where

$m_{r}=\frac{1}{n} \sum_{i=1}^{n}\left(x_{i}-\bar{x}\right)^{r}$

$s=\sqrt{m_{2}}$

Sample estimates of $\sqrt{\beta_{1}}$ and $\beta_{2}$ do not always coincide with the theoretical points or curves of their parent distributions, and methods of GOF test using $\left(\sqrt{b_{1}}, b_{2}\right)$ have thus been developed for the normal distribution (Fisher, 1930; D'Agostino and Pearson, 1973; Bowman and Shenton, 1975). Bowman and Shenton (1986) developed acceptance regions for a $\left(\sqrt{\beta_{1}}, \beta_{2}\right)$-based GOF test using stochastic simulation. The sample moments $\left(\sqrt{b_{1}}, b_{2}\right)$ tend to have high variances, especially when the sample sizes are small. Therefore, it is often difficult to distinguish among candidate distributions. Even though the joint distribution of the ordinary sample skewness and sample kurtosis is asymptotically normal, such asymptotic property is a poor approximation in small and moderately samples, particularly when the underlying distribution is even moderately skew (Hosking and Wallis, 1997). Using stochastic simulation, Wu (2005) studied the distribution of $\left(\sqrt{b_{1}}, b_{2}\right)$ for normal distribution with respect to sample sizes varying from 20 to 1000 . As can be seen in Fig. 2, for smaller sample sizes (for example $n=20$ ) the distribution of $\sqrt{b_{1}}$ given $b_{2}$ changes from unimodality to bimodality as $b_{2}$ increases. Thus, closed curves encompassing certain percentages of $\left(\sqrt{b_{1}}, b_{2}\right)$ samples of the normal distribution are difficult to be expressed by mathematical equations when the sample sizes are small.

\section{$L$-moments and the L-moment-ratio diagram}

L-moments are an alternative system of describing the shapes of probability distributions. For a random variable $X$ with quantile function $x(u)$, Hosking and Wallis (1997) defined the $L$-moments $\left(\lambda_{r}, r=1,2, \ldots\right)$ as

$\lambda_{r}=\int_{0}^{1} x(u) P_{r-1}^{*}(u) \mathrm{d} u$

where

$P_{r}^{*}(u)=\sum_{k=0}^{r} p_{r, k}^{*} u^{k}, \quad r=0,1,2, \ldots$

$p_{r, k}^{*}=(-1)^{r-k}\left(\begin{array}{c}r \\ k\end{array}\right)\left(\begin{array}{c}r+k \\ k\end{array}\right)=\frac{(-1)^{r-k}(r+k) !}{(k !)^{2}(r-k) !}$.
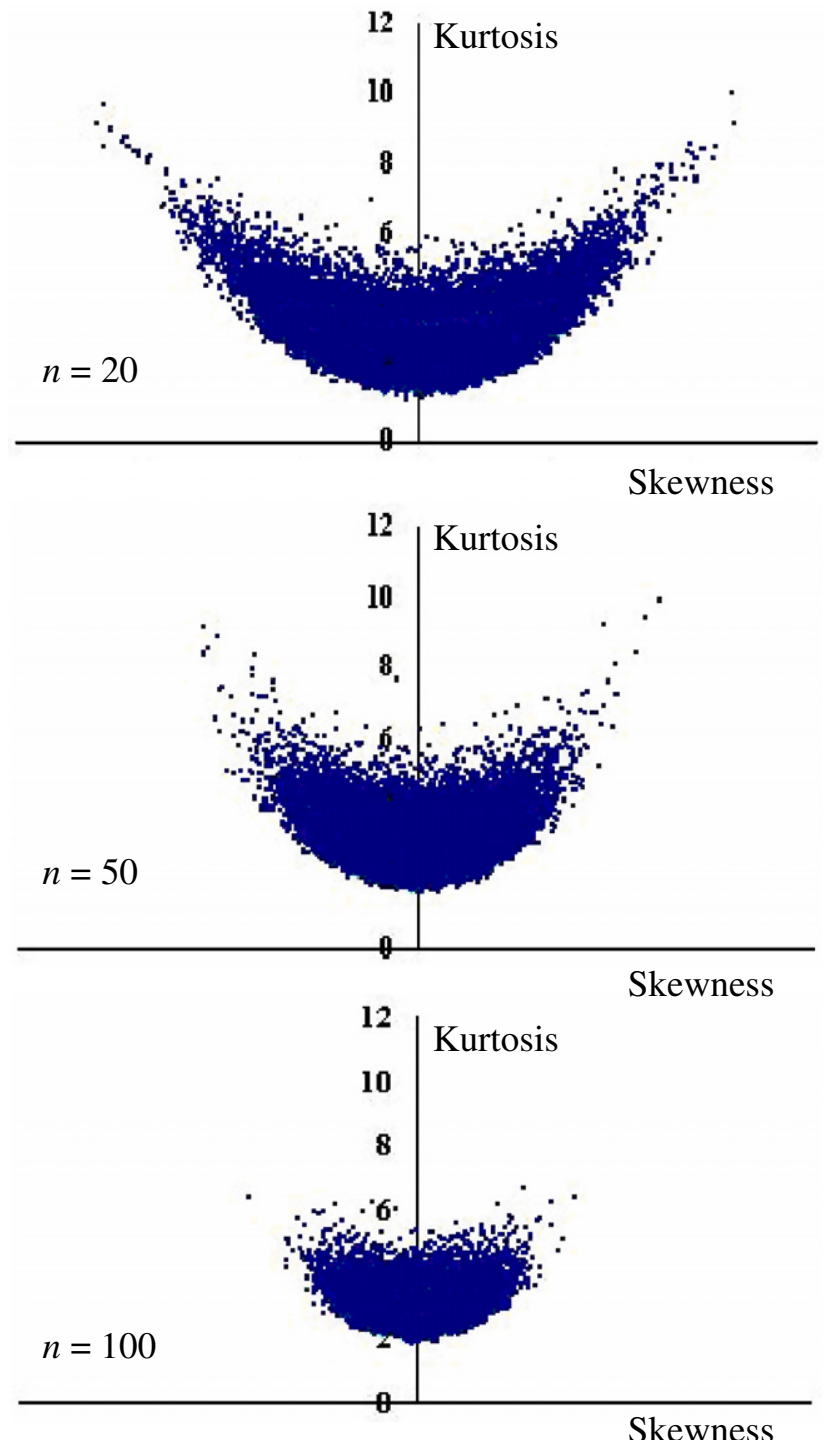

Figure 2 Scattering of sample moment ratios of the normal distribution (100,000 random samples). Values of $n$ represent size of the random samples for moments calculation ( $\mathrm{Wu}$, 2005).

The $L$-moments can also be expressed in terms of the probability-weighted moments defined by Greenwood et al. (1979), and the first four L-moments are given by

$$
\begin{aligned}
& \lambda_{1}=\beta_{0} \\
& \lambda_{2}=2 \beta_{1}-\beta_{0} \\
& \lambda_{3}=6 \beta_{2}-6 \beta_{1}+\beta_{0} \\
& \lambda_{4}=20 \beta_{3}-30 \beta_{2}+12 \beta_{1}-\beta_{0}
\end{aligned}
$$

where $\beta_{r}, r=0,1,2, \ldots$, are probability-weighted moments defined by

$\beta_{r}=\int_{0}^{1} x(u) u^{r} \mathrm{~d} u$

In terms of linear combination of order statistics, the L-moments can also be expressed by 
$\lambda_{1}=E\left(X_{1: 1}\right)$

$\lambda_{2}=\frac{1}{2} E\left(X_{2: 2}-X_{1: 2}\right)$

$\lambda_{3}=\frac{1}{3} E\left(X_{3: 3}-2 X_{2: 3}+X_{1: 3}\right)$

$\lambda_{4}=\frac{1}{4} E\left(X_{4: 4}-3 X_{3: 4}+3 X_{2: 4}-X_{1: 4}\right)$

where $X_{k: n}$ is the $k$ th order statistic from a random sample of size $n$.

Similar to the ordinary moment ratios, the $L$-moment ratios are defined by

$\tau_{r}=\frac{\lambda_{r}}{\lambda_{2}}, \quad r=3,4, \ldots$

Theoretical relationships between $L$-skewness $\left(\tau_{3}\right)$ and $L$ kurtosis $\left(\tau_{4}\right)$, i.e. the $L$-moment-ratio diagram, of several probability distributions have been given by Hosking (1990) and can be used to distinguish different probability distributions (see Fig. 3).

Given a random sample $\left\{x_{1}, x_{2}, \ldots, x_{n}\right\}$, an unbiased estimator of the probability-weighted moment $\beta_{r}$ is given by

$b_{r}=\frac{1}{n} \sum_{j=r+1}^{n} \frac{(j-1)(j-2) \cdots(j-r)}{(n-1)(n-2) \cdots(n-r)} x_{j: n}$

The sample $L$-moments $\left(\ell_{r}\right)$ and sample $L$-moment ratios $\left(t_{r}\right)$ can then be calculated by

$\ell_{1}=b_{0}$

$\ell_{2}=2 b_{1}-b_{0}$

$\ell_{3}=6 b_{2}-6 b_{1}+b_{0}$

$\ell_{4}=20 b_{3}-30 b_{2}+12 b_{1}-b_{0}$

$t_{r}=\ell_{r} / \ell_{2}$

The sample $L$-moments $\ell_{r}$ is an unbiased estimator of $\lambda_{r}$; however, the estimator $t_{r}$ is not an unbiased estimator of $\tau_{r}$ (Hosking and Wallis, 1997), even though for most distributions the biases are negligible for sample sizes of 20 or more. Also, the sample L-skewness $\left(t_{3}\right)$ and $L$-kurtosis $\left(t_{4}\right)$ are found to have a joint distribution close to bivariate nor-

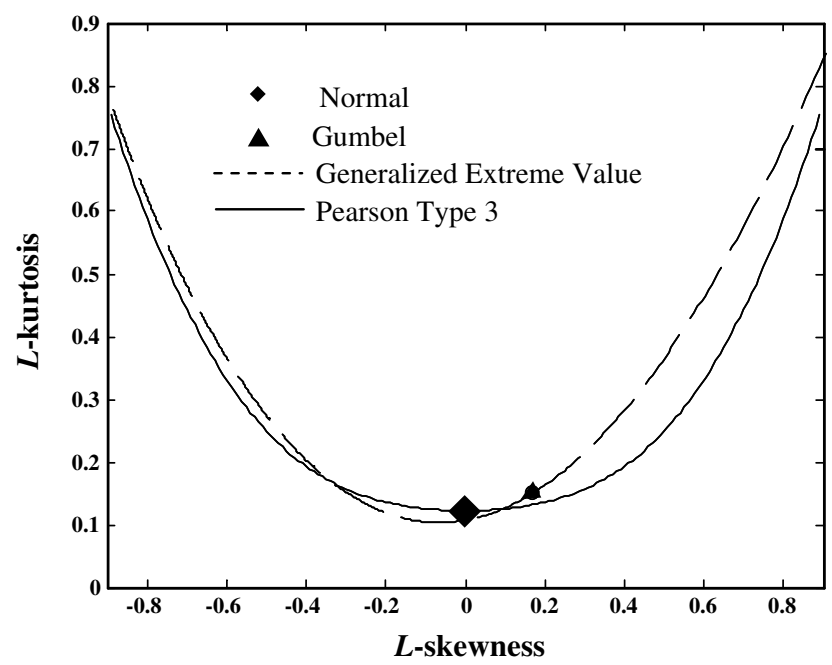

Figure 3 L-moment-ratio diagram of various distributions. mal. However, the exact distributions of the sample $L$-moment ratios are difficult to be derived.

In addition to the above mentioned sample $L$-moments and sample $L$-moment ratios, Hosking and Wallis (1997) also defined the plotting-position estimators of $\lambda_{r}$ and $\tau_{r}$ as

$\tilde{\lambda}_{r}=\frac{1}{n} \sum_{j=1}^{n} P_{r-1}^{*}\left(p_{j: n}\right) x_{j: n}$

$\tilde{\tau}_{r}=\tilde{\lambda}_{r} / \tilde{\lambda}_{2}$

where $p_{j: n}$ is a plotting-position estimator and was chosen to be

$p_{j: n}=(j-0.35) / n$.

Hosking and Wallis (1997) indicated that $\tilde{\lambda}_{r}$ is not an unbiased estimator of $\lambda_{r}$, but its bias tends to zero in large samples. Hereafter $t_{r}$ and $\tilde{\tau}_{r}$ will be, respectively, referred to as the probability-weighted-moment estimator and the plotting-position estimator of the $L$-moment ratio $\tau_{r}$. It is generally advised to use the probability-weighted-moment estimators since they are inferior to the plotting-position estimators only for some instances of estimation of extreme quantiles in regional frequency analysis and have generally lower bias as estimators of the $L$-moment ratios (Hosking and Wallis, 1997).

It should also be addressed that the plotting-position $L$ moment estimators are non-invariant estimators (Hosking and Wallis, 1997), and thus their statistical properties vary with changes in the location and scale parameters of the population from which random samples are drawn. In order to derive statistical properties of the plotting-position $L$ moment estimators which are generally applicable with respect to location and scale parameters, it is necessary to have the random samples preprocessed (sample mean subtraction followed by division by sample standard deviation) for normalization (zero mean and unit standard deviation), and use the standardized data for calculation of the plotting-position L-moment estimates using Eqs. (29) and (30).

\section{Stochastic simulation of normal and Gumbel distributions}

In this study, we chose the standard normal and standard Gumbel distributions (zero mean and unit standard deviation) to exemplify the proposed approach for construction of acceptance regions for GOF tests based on the L-moment-ratio diagram. $L$-moment ratios $\left(\tau_{3}, \tau_{4}\right)$ of the normal and Gumbel distributions are, respectively, $(0,0.1226)$ and $(0.1699,0.1504)$. Random number generators for many probability distributions such as the standard normal, uniform, Gumbel, and Pearson type III distributions can be found in mathematical or statistical softwares.

For either of the standard normal and standard Gumbel distribution, a total of 100,000 random samples were generated with respect to the specified sample size $n=20,30,40$, $50,60,75,100,150,250,500$, and 1000 . For each of the 100,000 samples, sample $L$-skewness and $L$-kurtosis were calculated using the probability-weighted-moment estimator (Eq. (28)) and the plotting-position estimator (Eq. (30)). Figs. 4 and 5, respectively, illustrate scattering of the sample $L$-skewness and $L$-kurtosis (for sample size 


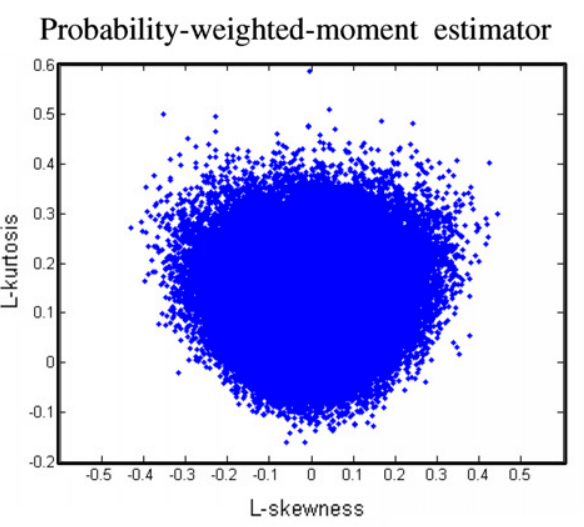

(a) $n=20$

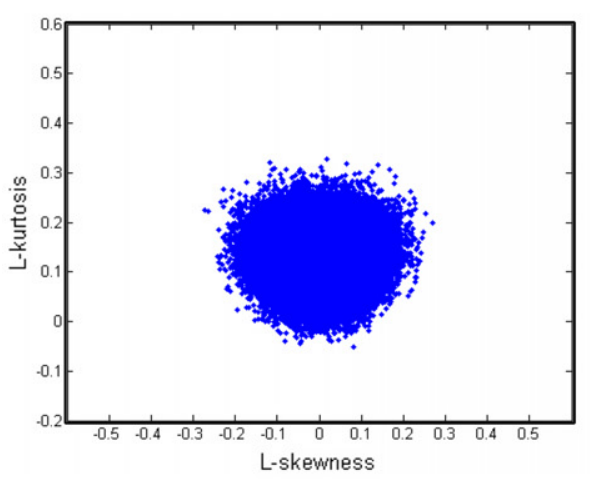

(b) $n=50$

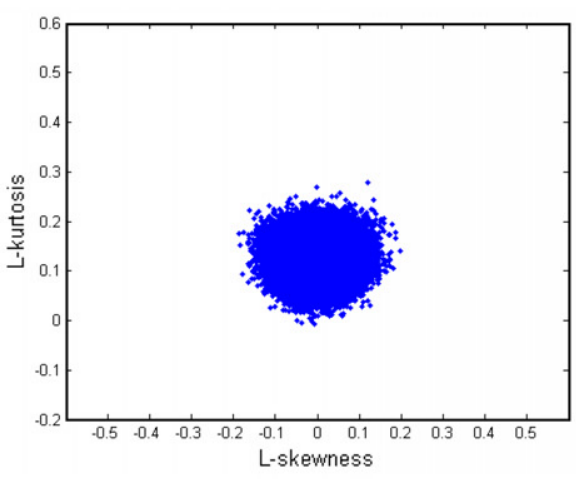

(c) $n=100$

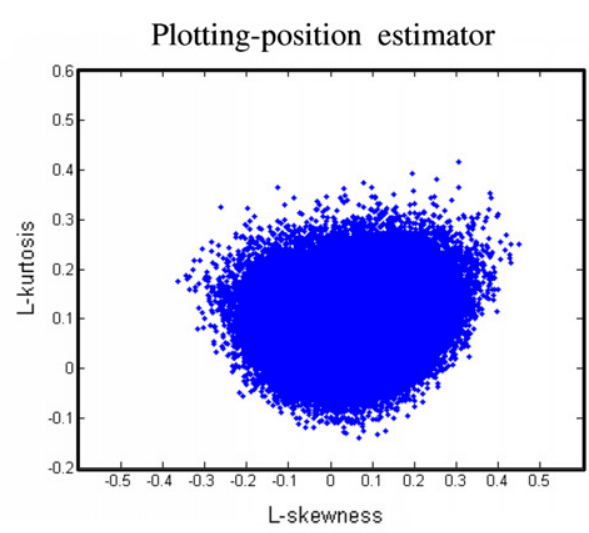

(d) $n=20$

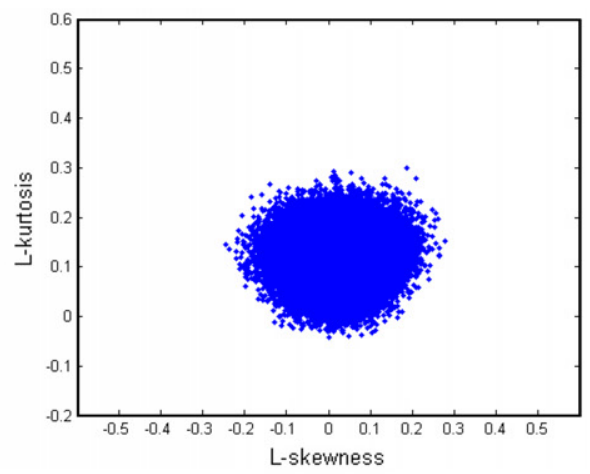

(e) $n=50$

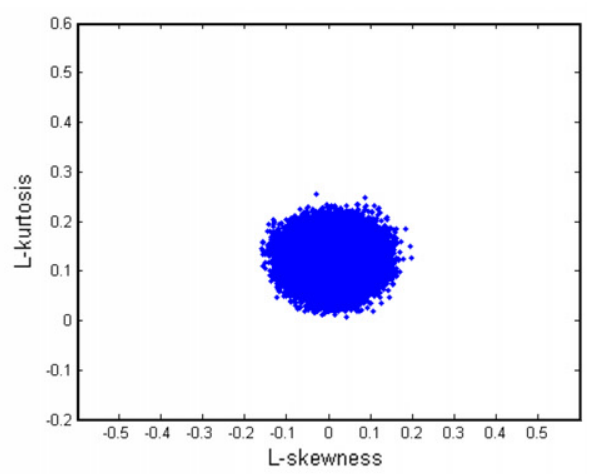

(f) $n=100$

Figure 4 Scattering of sample $L$-moment ratios of the normal distribution (100,000 random samples).

$n=20,50$ and 100 ) estimated by the probability-weightedmoment estimator and the plotting-position estimator using random samples of standard normal and Gumbel distributions. Readers are reminded that the plotting-position sample L-moments shown in Figs. 4 and 5 and used in subsequent analyses were calculated using standardized data as described in the last paragraph of ' $L$-moments and the $L$-moment-ratio diagram' section. It can be seen that, for both distribution types, the joint distribution of sample $L$-skewness and $L$-kurtosis seem to resemble a bivariate normal distribution for a larger sample size $(n=100)$. However, for sample size $n=20$, the joint distribution of sample $L$ skewness and $L$-kurtosis seems to differ from the bivariate normal. Particularly for Gumbel distribution, sample $L$-mo- ments of both estimators are positively skewed. For smaller sample sizes ( $n=20$ and 50), distribution clouds of the sample $L$-moment ratios estimated by the plotting-position method appear to have their centers located away from $\left(\tau_{3}, \tau_{4}\right)$, an indication of biased estimation. However, for sample size $n=100$, the bias is almost unnoticeable, suggesting that the bias in $L$-moment-ratio estimation using the plotting-position estimator is negligible for larger sample sizes. In contrast, distribution clouds of the sample $L$-moment ratios estimated by the probability-weighted-moment method appear to have their centers almost coincide with $\left(\tau_{3}, \tau_{4}\right)$.

Summary statistics based on the 100,000 sample estimates of $L$-skewness and $L$-kurtosis of the two distributions 


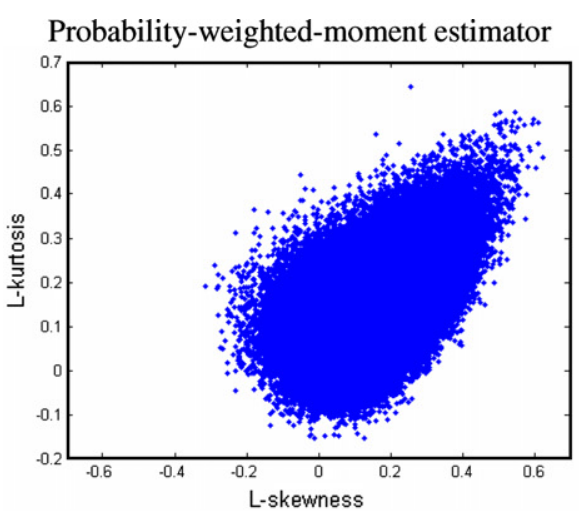

(a) $n=20$

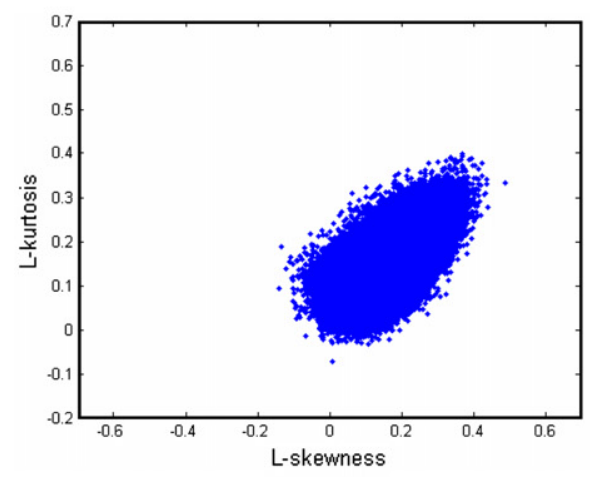

(b) $n=50$

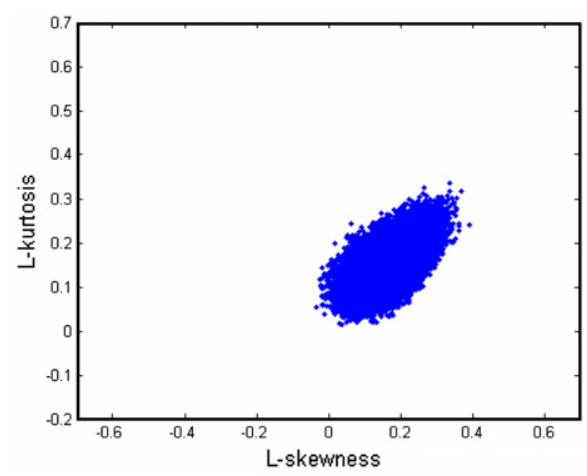

(c) $n=100$

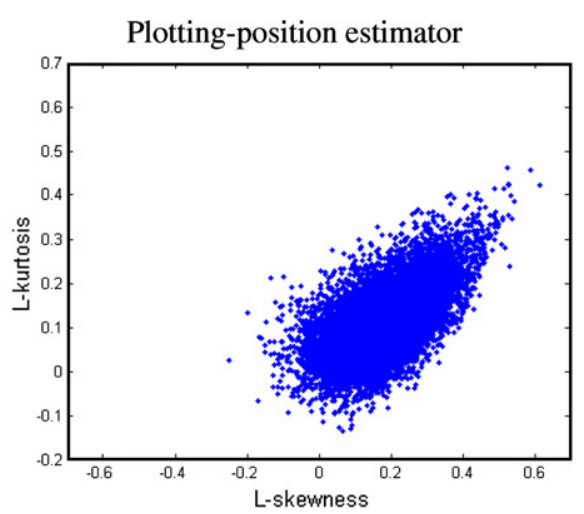

(d) $n=20$

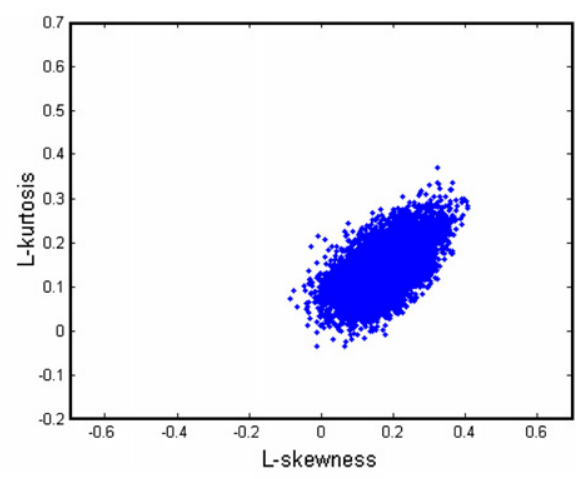

(e) $n=50$

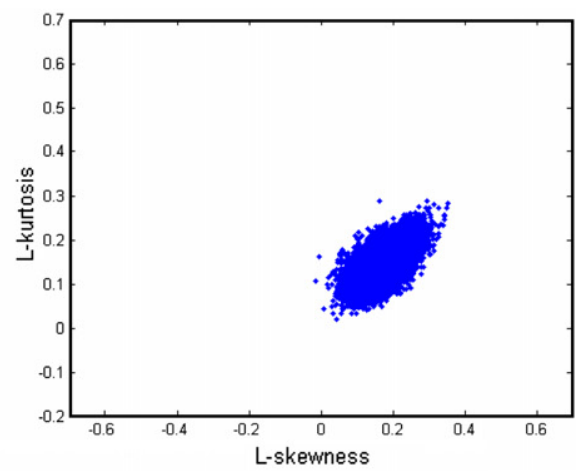

(f) $n=100$

Figure 5 Scattering of sample $L$-moment ratios of the Gumbel distribution $(100,000$ random samples).

are shown in Tables 1 and 2 . For convenience of explanation we will hereafter use the symbols $L-C S$ and $L-C K$ in subscript to represent properties of sample $L$-skewness and $L$-kurtosis, i.e. $\left(t_{3}, t_{4}\right)$ or $\left(\tilde{\tau}_{3}, \tilde{\tau}_{4}\right)$. For example, $\mu_{L-c s}$ represents the expected value of $t_{3}$ or $\tilde{\tau}_{3}$, and $\hat{\mu}_{L-c S}$ is the sample estimate of $\mu_{L \text {-cs }}$ using either the probability-weighted-moment estimator or the plotting-position estimator. According to Hosking (1990), for standard normal distribution $\left(\tau_{3}=0\right.$, $\tau_{4}=0.1226$ ) the asymptotic bias of $t_{4}$ is $0.03 / \mathrm{n}$. It is demonstrated in Fig. 6 that the bias of $t_{4}$, calculated using the 100,000 random samples with respect to various sample sizes $n$, is well characterized by the asymptotic curve of $0.03 / n$.
Using the data shown in Table 1 we also found that biases of the plotting-position estimators of the standard normal distribution, i.e. $\hat{\mu}_{L-c s}$ and $\hat{\mu}_{L-C K}-0.1226$, vary with the sample size $n$, and can be respectively approximated by $0.9042 / n$ and $-0.6639 / n$ (see Fig. 6). For the Gumbel distribution, biases of $t_{3}$ and $t_{4}$ are, respectively, approximated by $-0.1927 / n$ and $-0.0426 / n$, and biases of $\tilde{\tau}_{3}$ and $\tilde{\tau}_{4}$ are $\frac{0.3529}{n}+\frac{0.7705}{n^{2}}$ and $\frac{-0.5426}{n}-\frac{2.3331}{n^{2}}$, respectively (see Fig. 7). It can also be observed that even though the probability-weighted-moment estimators $\left(t_{3}\right.$ and $\left.t_{4}\right)$ perform better in terms of unbiasedness, the plotting-position estimators $\left(\tilde{\tau}_{3}\right.$ and $\left.\tilde{\tau}_{4}\right)$ have smaller variances as demonstrated in Fig. 8. 
Table 1 Summary statistics of estimates of $L$-skewness and $L$-kurtosis of standard normal distribution $\left(\tau_{3}=0, \tau_{4}=0.1226\right)$

\begin{tabular}{|c|c|c|c|c|c|c|}
\hline Sample size, $n$ & $\hat{\mu}_{L-C S}$ & $\hat{\mu}_{L-C K}$ & $\hat{\sigma}_{L-C S}^{2}$ & $\hat{\sigma}_{L-C S, L-C K}$ & $\hat{\sigma}_{L-C K}^{2}$ & $r^{\mathrm{a}}$ \\
\hline \multicolumn{7}{|c|}{ (a) Probability-weighted-moment estimator } \\
\hline 20 & 0.000336 & 0.123945 & 0.011550 & 0.000012 & 0.006717 & 0.001341 \\
\hline 30 & 0.000006 & 0.123977 & 0.007121 & 0.000030 & 0.003909 & 0.005765 \\
\hline 40 & 0.000248 & 0.123421 & 0.005191 & -0.000014 & 0.002711 & -0.003626 \\
\hline 50 & -0.000141 & 0.123155 & 0.004048 & -0.000010 & 0.002087 & -0.003595 \\
\hline 60 & 0.000030 & 0.123181 & 0.003351 & 0.000000 & 0.001678 & -0.000168 \\
\hline 75 & 0.000078 & 0.122938 & 0.002642 & 0.000001 & 0.001315 & 0.000661 \\
\hline 100 & 0.000175 & 0.123013 & 0.001959 & -0.000007 & 0.000962 & -0.004779 \\
\hline 150 & 0.000070 & 0.122767 & 0.001287 & -0.000005 & 0.000622 & -0.005108 \\
\hline 250 & 0.000036 & 0.122726 & 0.000759 & -0.000002 & 0.000364 & -0.004559 \\
\hline 500 & -0.000055 & 0.122644 & 0.000374 & 0.000000 & 0.000180 & -0.000989 \\
\hline 1000 & 0.000000 & 0.122601 & 0.000188 & 0.000000 & 0.000089 & -0.000618 \\
\hline \multicolumn{7}{|c|}{ (b) Plotting-position estimator } \\
\hline 20 & 0.045302 & 0.087778 & 0.009355 & 0.000710 & 0.003985 & 0.116248 \\
\hline 30 & 0.030006 & 0.101002 & 0.006203 & 0.000334 & 0.002776 & 0.080488 \\
\hline 40 & 0.022735 & 0.106699 & 0.004685 & 0.000164 & 0.002099 & 0.052397 \\
\hline 50 & 0.017865 & 0.110001 & 0.003730 & 0.000103 & 0.001703 & 0.040813 \\
\hline 60 & 0.015029 & 0.112337 & 0.003131 & 0.000078 & 0.001417 & 0.036998 \\
\hline 75 & 0.012076 & 0.114373 & 0.002503 & 0.000051 & 0.001149 & 0.030179 \\
\hline 100 & 0.009171 & 0.116656 & 0.001881 & 0.000022 & 0.000870 & 0.017280 \\
\hline 150 & 0.006069 & 0.118583 & 0.001253 & 0.000008 & 0.000581 & 0.009574 \\
\hline 250 & 0.003635 & 0.120239 & 0.000747 & 0.000002 & 0.000350 & 0.004205 \\
\hline 500 & 0.001745 & 0.121409 & 0.000371 & 0.000001 & 0.000177 & 0.003359 \\
\hline 1000 & 0.000900 & 0.121986 & 0.000187 & 0.000000 & 0.000088 & 0.001568 \\
\hline
\end{tabular}

${ }^{a}$ Correlation coefficient of estimates of $L$-skewness and $L$-kurtosis.

\section{Evaluating the bivariate normality assumption for sample $L$-skewness and $L$-kurtosis using the Mardia test}

Even though the exact distributions of sample $L$-moments are difficult to derive, constructing the acceptance regions for L-moments-based GOF test using a tractable distribution is often desired and of practical usage. Figs. 4 and 5 show that distribution clouds of $\left(t_{3}, t_{4}\right)$ and $\left(\tilde{\tau}_{3}, \tilde{\tau}_{4}\right)$ resemble a bivariate normal distribution visually for larger sample sizes. Thus, the bivariate normality for sample $L$-skewness and $L$-kurtosis is a convenient assumption in our study and its adequacy with respect to different sample sizes is evaluated by way of a goodness-of-fit test for bivariate normality.

There have been a few methods of goodness-of-fit test for multivariate normality in the literature. Cox (1968) and Andrews et al. (1973) recommended an informal graphical procedure for assessing multivariate normality. Mardia (1970) defined measures for multivariate skewness and kurtosis and developed two test statistics for test of bivariate normality. Cox and Small (1978), considering the linearity of regression relations between two jointly distributed normal random variables, developed a test statistic of quadratic form which has a chi-squares distribution with two degree of freedom. The Mardia (1970) test briefly described below is applied in this study.

Let $X^{\top}=\left(X_{1}, X_{2}, \ldots, X_{p}\right)$ be a $p$-variate random vector. Given a random sample of $X$ of size $N$, Mardia defined the fol- lowing two estimators for measures of multivariate skewness $\left(b_{1, p}\right)$ and kurtosis $\left(b_{2, p}\right)$

$$
\begin{aligned}
& b_{1, p}=\frac{1}{N} \sum_{i=1}^{N} \sum_{j=1}^{N}\left[\left(X_{i}-\bar{X}\right)^{\top} S^{-1}\left(X_{j}-\bar{X}\right)\right]^{3} \\
& b_{2, p}=\frac{1}{N} \sum_{i=1}^{N}\left[\left(X_{i}-\bar{X}\right)^{\top} S^{-1}\left(X_{i}-\bar{X}\right)\right]^{2}
\end{aligned}
$$

where $\bar{X}$ and $S$, respectively, are the sample mean vector and the sample covariance matrix of $X$. For a random sample of multivariate normal distribution, it can be shown that $N b_{1, p} / 6$ has an asymptotic $\chi^{2}$ distribution with $p(p+1)(p+2) / 6$ degrees of freedom, and $b_{2, p}$ has an asymptotic normal distribution with mean $p(p+2)(N-1) /(N+1)$ and variance $8 p(p+2) / N$ (Mardia, 1970). Hence, the following Mardia test statistic $M_{2, p}$ has an asymptotic standard normal distribution

$M_{2, p}=\frac{b_{2, p}-[p(p+2)(N-1) /(N+1)]}{\sqrt{8 p(p+2) / N}}$.

At level of significance $\alpha$, the null hypothesis of multivariate normality is rejected if

$\left|M_{2, p}\right|>z_{1-\alpha / 2}$

where $z_{1-\alpha / 2}$ is the $100(1-\alpha / 2) \%$ quantile of the standard normal distribution. In our study, the level of significance $\alpha$ is chosen to be 0.05 . Hence, the null hypothesis is rejected if the absolute value of the Mardia statistic $M_{2, p}$ exceeds 1.96 . 
Table 2 Summary statistics of estimates of $L$-skewness and $L$-kurtosis of standard Gumbel distribution $\left(\tau_{3}=0.1699, \tau_{4}=0.1504\right)$

\begin{tabular}{|c|c|c|c|c|c|c|}
\hline Sample size, $n$ & $\hat{\mu}_{L-C S}$ & $\hat{\mu}_{L-C K}$ & $\hat{\sigma}_{L-C S}^{2}$ & $\hat{\sigma}_{L-C S, L-C K}$ & $\hat{\sigma}_{L-\mathrm{CK}}^{2}$ & $r^{a}$ \\
\hline \multicolumn{7}{|c|}{ (a) Probability-weighted-moment estimator } \\
\hline 20 & 0.160062 & 0.148051 & 0.013281 & 0.005802 & 0.009028 & 0.529844 \\
\hline 30 & 0.163816 & 0.149323 & 0.008512 & 0.003803 & 0.005480 & 0.556854 \\
\hline 40 & 0.164726 & 0.149240 & 0.006251 & 0.002781 & 0.003890 & 0.564091 \\
\hline 50 & 0.166199 & 0.149461 & 0.004906 & 0.002223 & 0.003032 & 0.576329 \\
\hline 60 & 0.166703 & 0.149803 & 0.004050 & 0.001834 & 0.002477 & 0.579178 \\
\hline 75 & 0.167600 & 0.149915 & 0.003214 & 0.001476 & 0.001969 & 0.586935 \\
\hline 100 & 0.168106 & 0.150102 & 0.002380 & 0.001100 & 0.001449 & 0.592177 \\
\hline 150 & 0.168521 & 0.150052 & 0.001573 & 0.000728 & 0.000949 & 0.595811 \\
\hline 250 & 0.169147 & 0.150209 & 0.000941 & 0.000437 & 0.000563 & 0.600204 \\
\hline 500 & 0.169484 & 0.150203 & 0.000467 & 0.000219 & 0.000278 & 0.606975 \\
\hline 1000 & 0.169764 & 0.150350 & 0.000231 & 0.000107 & 0.000138 & 0.601309 \\
\hline \multicolumn{7}{|c|}{ (b) Plotting-position estimator } \\
\hline 20 & 0.189560 & 0.117413 & 0.010833 & 0.004853 & 0.006022 & 0.600780 \\
\hline 30 & 0.182364 & 0.129767 & 0.007388 & 0.003341 & 0.004180 & 0.601194 \\
\hline 40 & 0.179440 & 0.135395 & 0.005633 & 0.002562 & 0.003216 & 0.601861 \\
\hline 50 & 0.177144 & 0.138612 & 0.004495 & 0.002052 & 0.002584 & 0.602147 \\
\hline 60 & 0.176168 & 0.140700 & 0.003773 & 0.001727 & 0.002184 & 0.601496 \\
\hline 75 & 0.174656 & 0.142748 & 0.003051 & 0.001409 & 0.001774 & 0.605611 \\
\hline 100 & 0.173681 & 0.144721 & 0.002300 & 0.001064 & 0.001340 & 0.605962 \\
\hline 150 & 0.172428 & 0.146622 & 0.001537 & 0.000717 & 0.000907 & 0.607566 \\
\hline 250 & 0.171318 & 0.148192 & 0.000925 & 0.000429 & 0.000544 & 0.604384 \\
\hline 500 & 0.170603 & 0.149251 & 0.000465 & 0.000216 & 0.000274 & 0.606725 \\
\hline 1000 & 0.170246 & 0.149807 & 0.000233 & 0.000108 & 0.000137 & 0.605377 \\
\hline
\end{tabular}

a Correlation coefficient of estimates of $L$-skewness and $L$-kurtosis.

Results of the Mardia test for bivariate normality of $\left(t_{3}, t_{4}\right)$ and $\left(\tilde{\tau}_{3}, \tilde{\tau}_{4}\right)$ of the normal and Gumbel distributions are illustrated in Fig. 9. It appears that the assumption of bivariate normal distribution for sample $L$-skewness and $L$ kurtosis of both distributions is valid for moderate to large sample sizes. However, for random samples of normal distribution with sample size $n \leqslant 30$, the bivariate normal assumption may not be adequate. Similarly, the bivariate normal assumption for sample $L$-skewness and $L$-kurtosis of the Gumbel distribution may not be adequate for sample size $n \leqslant 60$.

\section{Establishing acceptance regions for GOF tests}

We have demonstrated that, for moderate to large sample sizes, the sample $L$-skewness and $L$-kurtosis of both the normal and Gumbel distributions have asymptotic bivariate normal distributions. Using this property, the $100(1-\alpha) \%$ acceptance region of a GOF test based on sample $L$-skewness and $L$-kurtosis can be determined by the equiprobable density contour of the bivariate normal distribution with its encompassing area equivalent to $1-\alpha$. Construction of such equiprobable contours is described below.

The probability density function of a multivariate normal distribution is generally expressed by

$f(X)=\frac{1}{(2 \pi)^{\frac{p}{2}}|\Sigma|^{\frac{1}{2}}} \mathrm{e}^{-\frac{1}{2}(X-\mu)^{\top} \Sigma^{-1}(X-\mu)}$

where $X$ is the $p$-variate random vector and $\mu$ and $\Sigma$, respectively, represent the mean vector and covariance matrix of
$X$. In the above equation the probability density function depends on the random vector $X$ only through the quadratic form $Q=(X-\mu)^{\top} \Sigma^{-1}(X-\mu)$ which has a chi-square distribution with $p$ degrees of freedom. Therefore, probability density contours of a multivariate normal distribution can be expressed by

$Q=(X-\mu)^{\top} \Sigma^{-1}(X-\mu)=c$

for any constant $c \geqslant 0$. For a bivariate normal distribution $(p=2)$, Eq. (37) represents an equiprobable ellipse, and a set of equiprobable ellipses can be constructed by assigning $\chi_{2, \alpha}^{2}$ to $c$ for various values of $\alpha$. Consequently, the $100(1-\alpha) \%$ acceptance region of a GOF test based on the sample $L$-skewness and $L$-kurtosis, i.e. $X^{\top}=\left(t_{3}, t_{4}\right)$ or $\left(\tilde{\tau}_{3}, \tilde{\tau}_{4}\right)$, is expressed by

$(X-\mu)^{\top} \Sigma^{-1}(X-\mu) \leqslant \chi_{2, \alpha}^{2}$

where $\chi_{2, \alpha}^{2}$ is the upper quantile of the $\chi_{2}^{2}$ distribution at significance level $\alpha$. For $c=1$ in Eq. (37), the ellipse has its center located at $\mu$, the half-lengths of its major and minor axes respectively equal to square root of the eigenvalues of $\Sigma$, and the rotation angle $\phi$ between its major axis and the horizontal axis equal to the vector angle of the maximum eigenvector (Heiberger and Holland, 2004).

For bivariate normal random vector $X^{\top}=\left(X_{1} X_{2}\right)$, the mean vector and the covariance matrix are, respectively,

$\mu=\left(\begin{array}{l}\mu_{1} \\ \mu_{2}\end{array}\right)$

and 

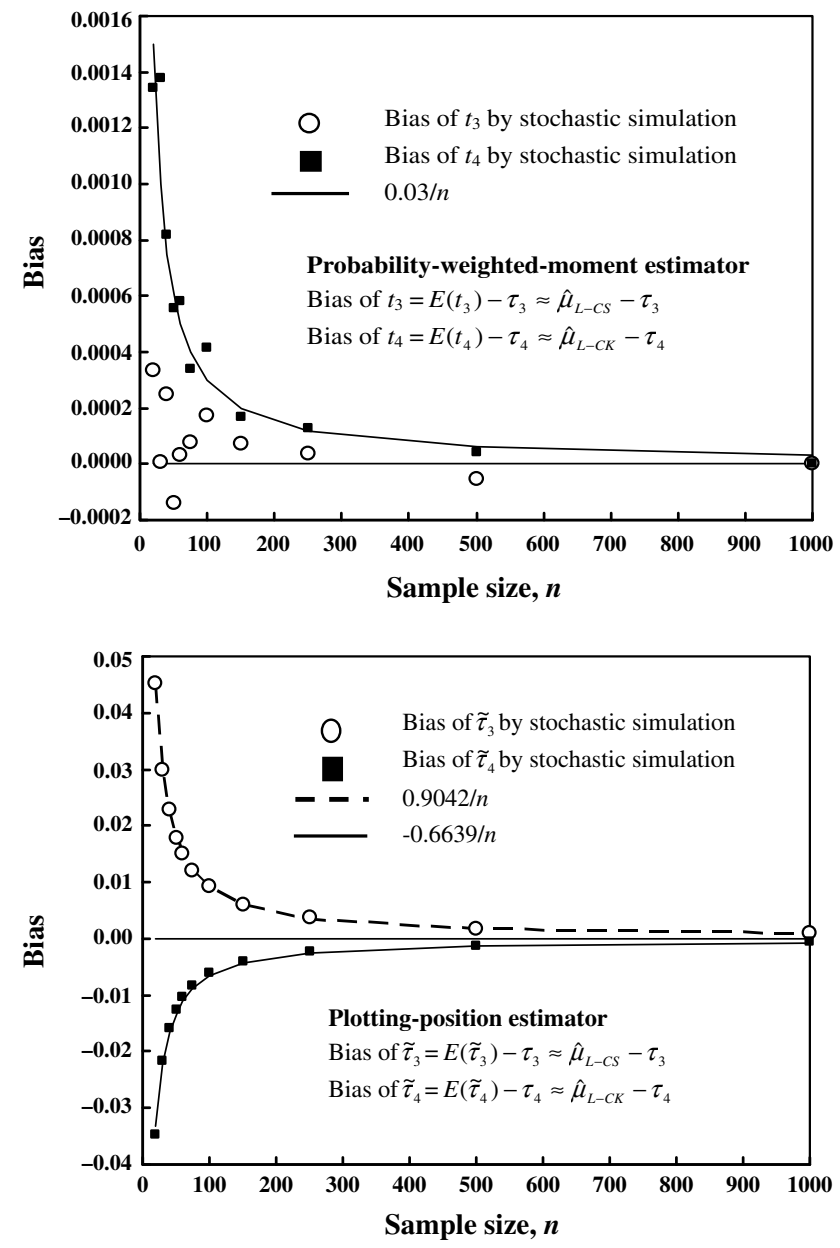

Figure 6 Bias of sample $L$-skewness and $L$-kurtosis of normal distribution.

$$
\Sigma=\left[\begin{array}{cc}
\operatorname{Var}\left(X_{1}\right) & \operatorname{Cov}\left(X_{1}, X_{2}\right) \\
\operatorname{Cov}\left(X_{1}, X_{2}\right) & \operatorname{Var}\left(X_{2}\right)
\end{array}\right]=\left[\begin{array}{cc}
\sigma_{1}^{2} & \rho \sigma_{1} \sigma_{2} \\
\rho \sigma_{1} \sigma_{2} & \sigma_{2}^{2}
\end{array}\right]
$$

where $\sigma_{1}$ and $\sigma_{2}$ are, respectively, standard deviation of $X_{1}$ and $X_{2}$, and $\rho$ is the correlation coefficient between $X_{1}$ and $X_{2}$. Hence, the density contour of $(X-\mu)^{\top} \Sigma^{-1}(X-\mu)=c$ can also be expressed by

$$
\frac{1}{1-\rho^{2}}\left[\frac{\left(X_{1}-\mu_{1}\right)^{2}}{\sigma_{1}^{2}}-\frac{2 \rho\left(X_{1}-\mu_{1}\right)\left(X_{2}-\mu_{2}\right)}{\sigma_{1} \sigma_{2}}+\frac{\left(X_{2}-\mu_{2}\right)^{2}}{\sigma_{2}^{2}}\right]=c
$$

Or equivalently, through the following coordinate shifting and rotation,

$$
\left(\begin{array}{l}
Y_{1} \\
Y_{2}
\end{array}\right)=\left[\begin{array}{cc}
\cos \phi & \sin \phi \\
-\sin \phi & \cos \phi
\end{array}\right] \cdot\left(\begin{array}{c}
X_{1}-\mu_{1} \\
X_{2}-\mu_{2}
\end{array}\right)
$$

the equiprobable ellipse can be expressed as

$$
\left(\frac{Y_{1}}{\sqrt{\lambda_{1} c}}\right)^{2}+\left(\frac{Y_{2}}{\sqrt{\lambda_{2} c}}\right)^{2}=1
$$

where $\lambda_{1}$ and $\lambda_{2}$ are eigenvalues of $\Sigma$ and $\left(Y_{1} Y_{2}\right)$ is the new coordinate after shifting and rotation of the original coordinate $\left(X_{1} X_{2}\right)$ by Eq. (42). It can also be shown (Mardia et al.,
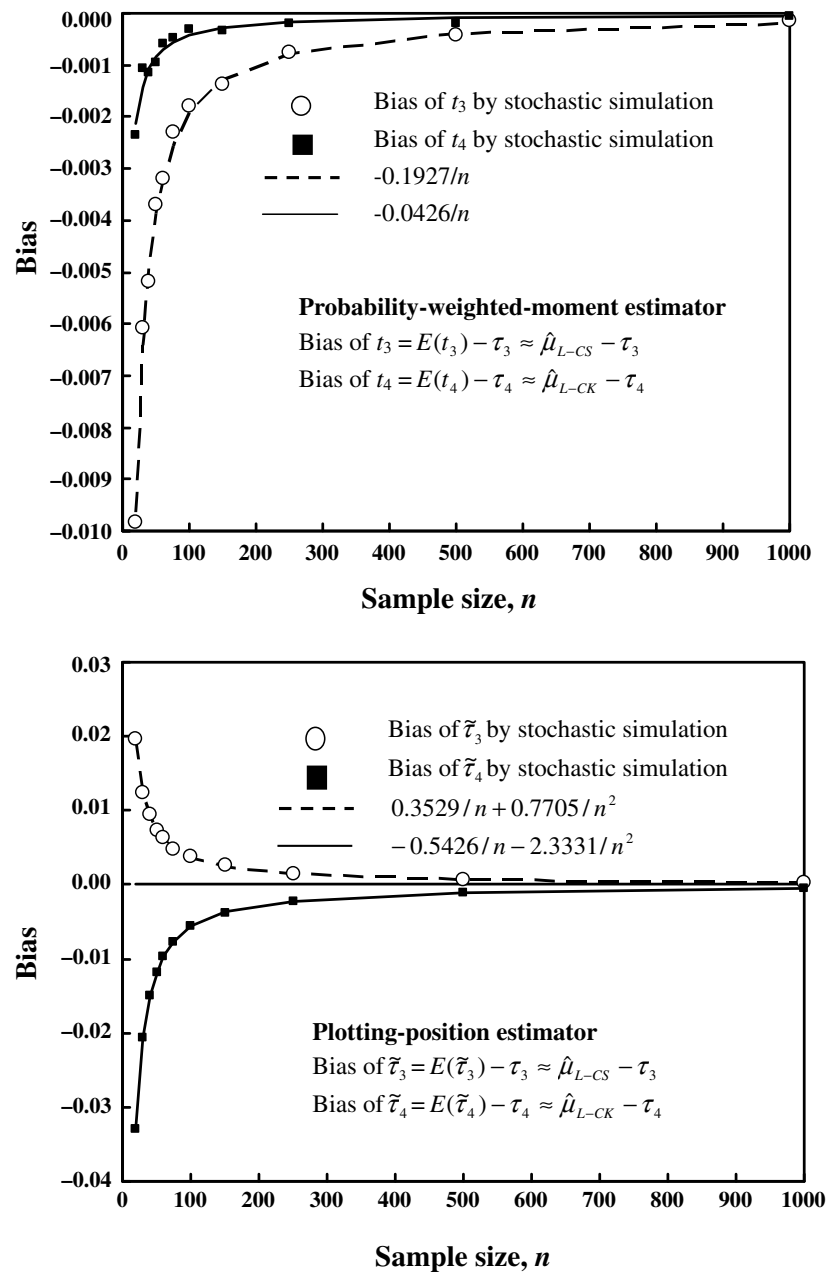

Figure 7 Bias of sample $L$-skewness and $L$-kurtosis of Gumbel distribution.

1979) that for the bivariate case the rotation angle $\phi$ is given by

$\phi=\tan ^{-1}\left(\frac{\lambda_{1}-\sigma_{1}^{2}}{\rho \sigma_{1} \sigma_{2}}\right)$

However, the expected values and covariance matrix of sample $L$-skewness and $L$-kurtosis are unknown and can only be estimated from random samples generated by stochastic simulation. Thus, in construction of the equiprobable ellipses, population parameters $\mu, \Sigma$, and $\rho$ must be, respectively, replaced by their sample estimates $\bar{x}, S$, and $r$. The term $Q$ in Eq. (37) therefore becomes the well-known Hotelling's $T^{2}$ statistic

$$
\begin{aligned}
T^{2} & =(X-\overline{\boldsymbol{x}})^{T} S^{-1}(X-\overline{\boldsymbol{x}}) \\
& =\frac{1}{1-r^{2}}\left[\frac{\left(X_{1}-\overline{\boldsymbol{x}}_{1}\right)^{2}}{s_{1}^{2}}-\frac{2 r\left(X_{1}-\overline{\boldsymbol{x}}_{1}\right)\left(X_{2}-\overline{\boldsymbol{X}}_{2}\right)}{s_{1} s_{2}}+\frac{\left(X_{2}-\overline{\boldsymbol{X}}_{2}\right)^{2}}{s_{2}^{2}}\right]
\end{aligned}
$$

where $s_{1}^{2}$ and $s_{2}^{2}$ represent the unbiased sample variances. Unlike $Q$ in Eq. (37) which has a chi-square distribution, the Hotelling's $T^{2}$ is distributed as a multiple of an F-distribution, i.e., 

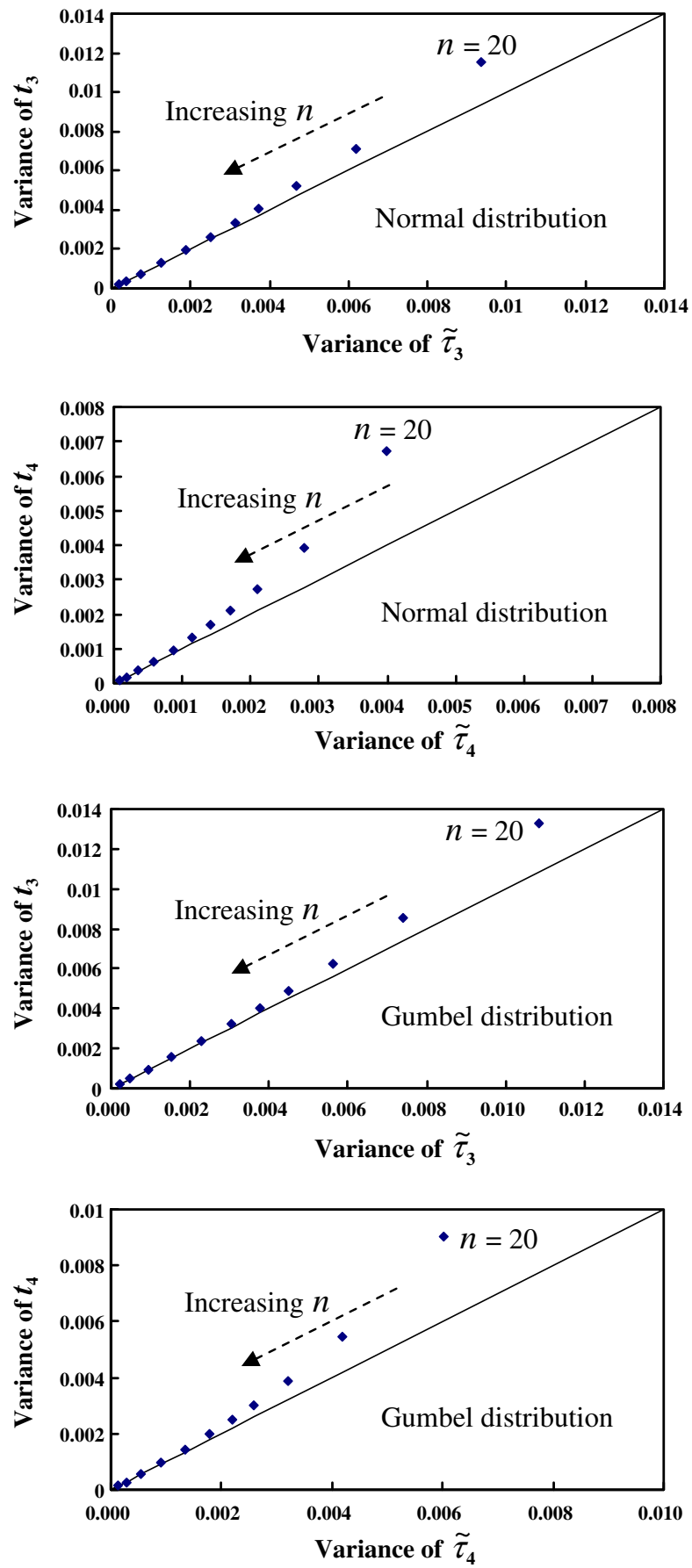

Figure 8 Comparison of variances of sample $L$-skewness and $L$-kurtosis estimated by the probability-weighted-moment estimator and the plotting-position estimator with respect to different values of sample size $n$ (see data in Tables 1 and 2).

$T^{2} \sim \frac{2\left(N^{2}-1\right)}{N(N-2)} F_{(2, N-2)}$

where $N$ is the size of a random sample of the bivariate vector $X$.

Using Eqs. (45) and (46), the $100(1-\alpha) \%$ confidence intervals of $L$-skewness and $L$-kurtosis can be determined. Such confidence intervals are dependent on $\bar{x}, S$, and $N$, the sample size of the sample $L$-moments pair. However,
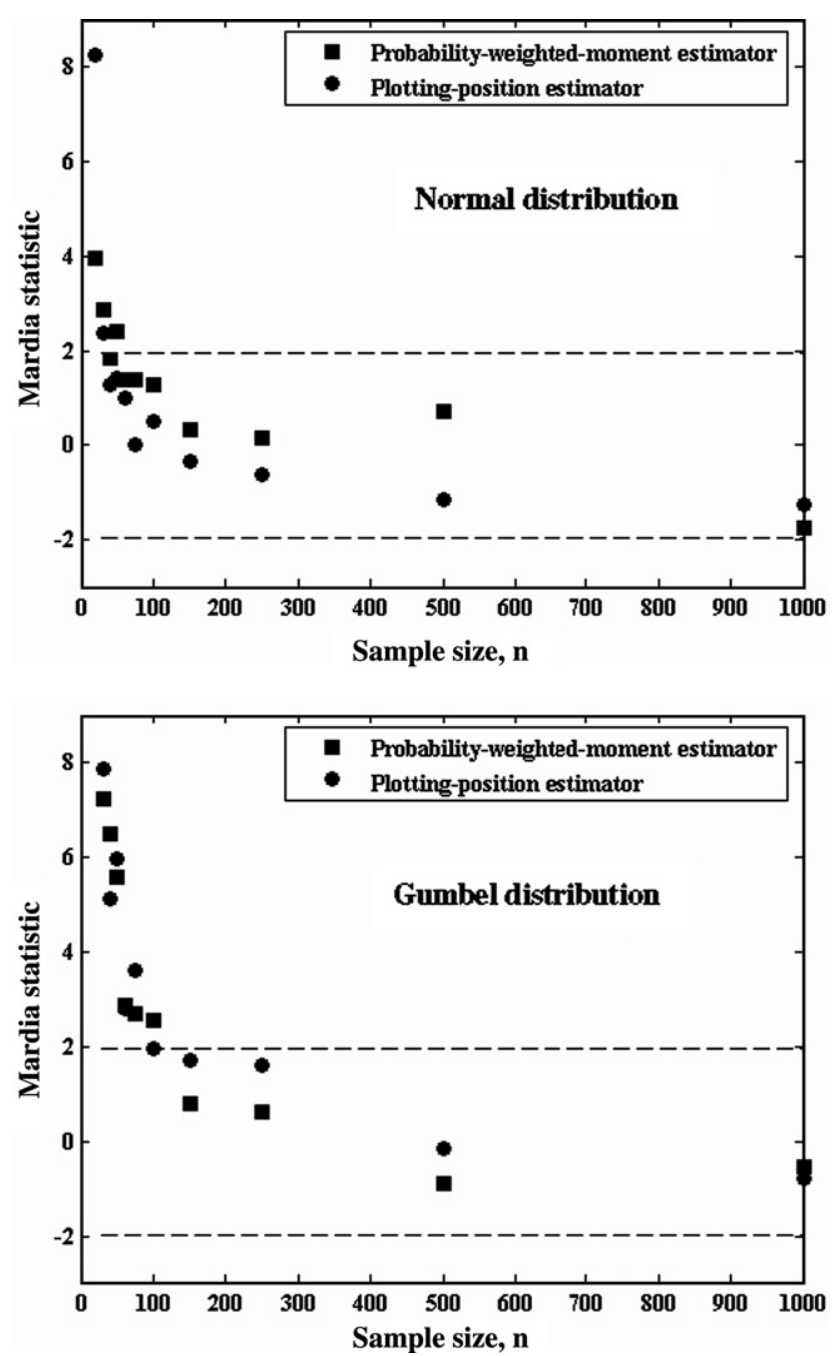

Figure 9 Results of the Mardia test for bivariate normality of sample $L$-skewness and $L$-kurtosis of the normal and Gumbel distributions.

the $100(1-\alpha) \%$ acceptance region of a GOF test represents a fixed area covering $100(1-\alpha) \%$ of the sample space. In our study a total of 100,000 realizations were generated for a given sample size $n$, yielding 100,000 pairs of sample $L$-skewness and $L$-kurtosis. At $N=100,000$, we have

$\frac{2\left(N^{2}-1\right)}{N(N-2)} F_{2, N-2}(\alpha) \approx 2 F_{2, N-2}(\alpha)=\chi_{2, \alpha}^{2}$

Therefore, the distribution of the Hotelling's $T^{2}$ can be well approximated by the chi-square distribution with degree of freedom 2 . This can also be explained by the fact that with very large sample size $(N=100,000)$ we can expect estimates of the mean and covariance matrix of sample $L$-skewness and $L$-kurtosis to be very close to their corresponding distribution parameters and hence $T^{2}$ is almost the same as $Q$ in Eq. (37).

Thus, if the sample $L$-moments pair, $\left(t_{3}, t_{4}\right)$ or $\left(\tilde{\tau}_{3}, \tilde{\tau}_{4}\right)$, of a random sample of size $n$ falls outside of the corresponding ellipse, i.e.

$T^{2}=\left(X-\bar{x}_{n}\right)^{\top} S_{n}^{-1}\left(X-\bar{x}_{n}\right)>\chi_{2, \alpha}^{2}$ 
the null hypothesis that the random sample is originated from a normal or Gumbel distribution is rejected. To avoid confusion, the sample mean $\left(\bar{x}_{n}\right)$ and covariance matrix $\left(S_{n}\right)$ of the bivariate vector $X$ in the above equation have been expressed with a subscript $n$ to indicate the size of the random sample from which sample $L$-moments were calculated. It is worthy to emphasize that the value of $N$ in Eqs. (34) and (46) represents the sample size of the bivariate vector $X$ and equals 100,000 in our study. It is not the

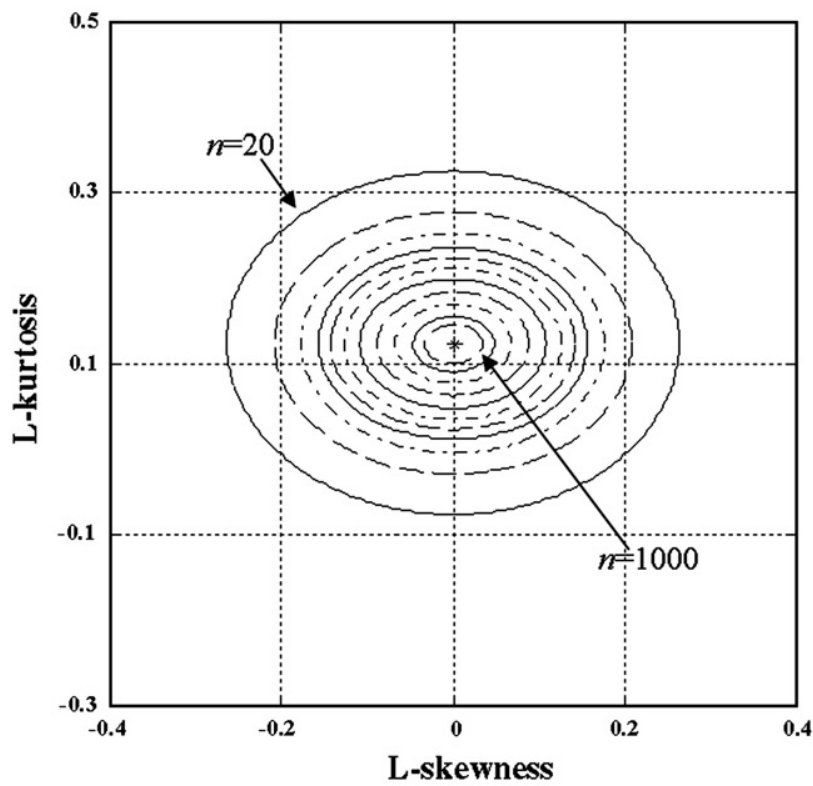

(a) Probability-weighted-moment estimator $\left(t_{3}, t_{4}\right)$

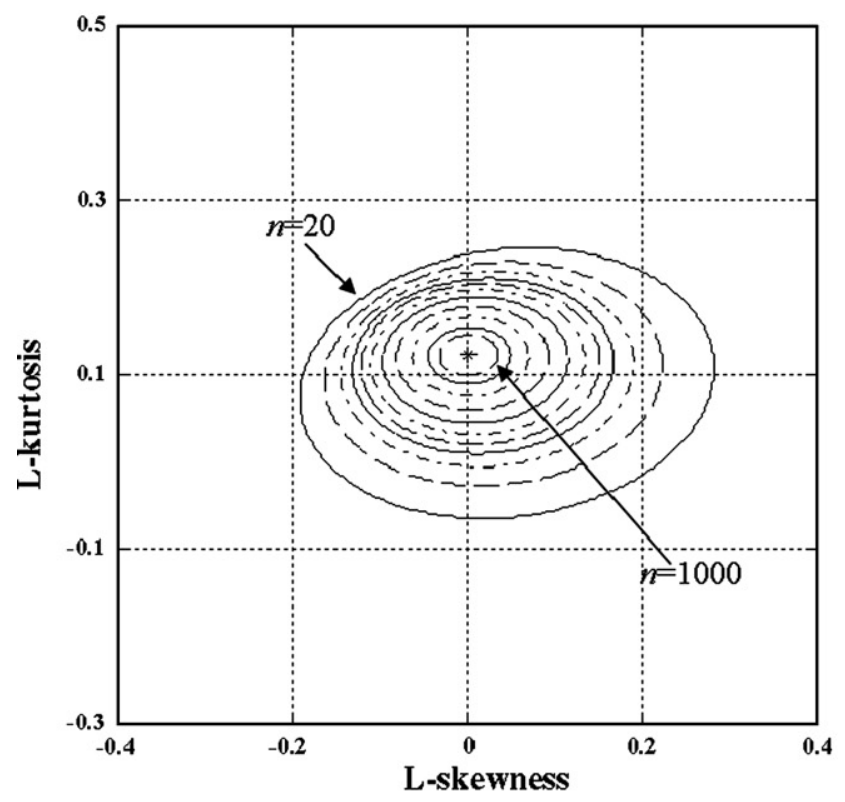

(b) Plotting-position estimator $\left(\tilde{\tau}_{3}, \widetilde{\tau}_{4}\right)$.

Figure 10 Ninety-five percent acceptance regions of $L$ moments-based GOF test for the normal distribution $\left(\tau_{3}=0, \tau_{4}=0.1226\right)$. Acceptance ellipses correspond to various sample sizes $(n=20,30,40,50,60,75,100,150,250,500$, and 1000). same as the sample size $n$ of a random sample of the normal or Gumbel random variable $X$ in 'Stochastic simulation of normal and Gumbel distributions' section.

As demonstrated in Figs. 6-8, the bias and sample variances of $\left(t_{3}, t_{4}\right)$ and $\left(\tilde{\tau}_{3}, \tilde{\tau}_{4}\right)$ reduce with the increase of sample size $n$, and therefore, a set of sample-size-dependent $95 \%$ acceptance regions can be constructed. Figs. 10 and 11 demonstrate the sample-size-dependent 95\% acceptance regions of GOF tests using $\left(t_{3}, t_{4}\right)$ and $\left(\tilde{\tau}_{3}, \tilde{\tau}_{4}\right)$ for the normal

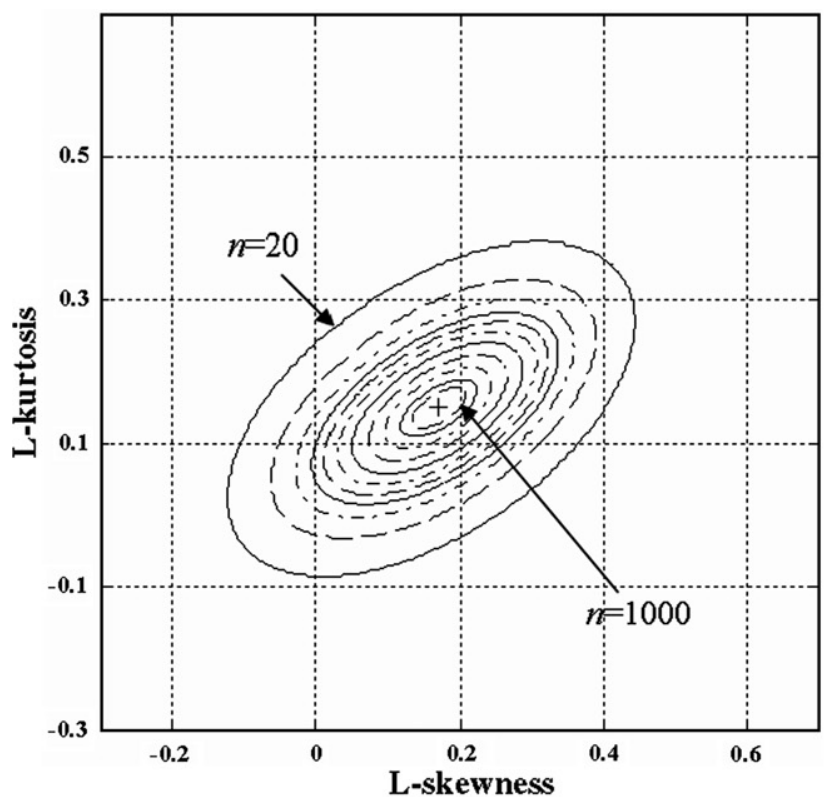

(a) Probability-weighted-moment estimator $\left(t_{3}, t_{4}\right)$

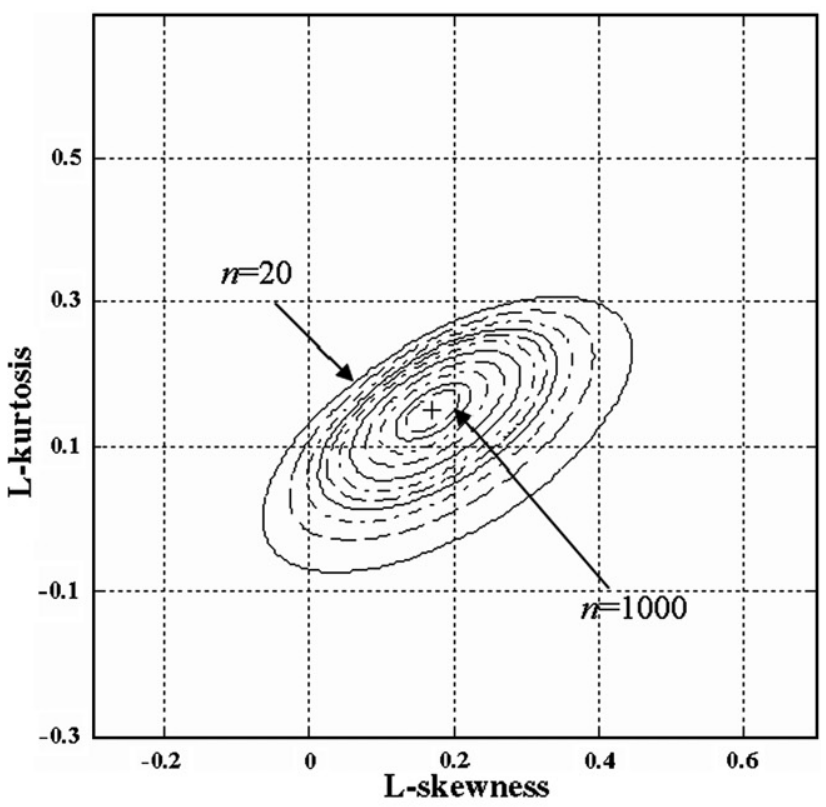

(b) Plotting-position estimator $\left(\tilde{\tau}_{3}, \widetilde{\tau}_{4}\right)$.

Figure 11 Ninety-five percent acceptance regions of $L$ moments-based GOF test for the Gumbel distribution $\left(\tau_{3}=0.1699, \tau_{4}=0.1504\right)$. Acceptance ellipses correspond to various sample sizes $(n=20,30,40,50,60,75,100,150,250$, 500 , and 1000). 
and Gumbel distributions, respectively. The bias in L-moment-ratios estimation by the plotting-position estimator $\left(\tilde{\tau}_{3}, \tilde{\tau}_{4}\right)$ is apparent for both distributions, whereas the bias by the probability-weighted-moment estimator $\left(t_{3}, t_{4}\right)$ is almost unnoticeable. As the sample size $n$ increases, the $95 \%$ acceptance regions shrink toward the theoretical L-moment-ratios, and the bias in $L$-moment-ratios estimation becomes negligible for both the normal and the Gumbel distributions.

\section{Empirical relationships between parameters of acceptance regions and sample size}

Since the $95 \%$ acceptance regions of the proposed GOF tests are dependent on the sample size $n$, it is therefore worthy to investigate the feasibility of establishing empirical relationships between the $95 \%$ acceptance region and the sample size. Such empirical relationships can be established using the following regression model

$\hat{\theta}(n)=\frac{a}{n}+\frac{b}{n^{2}}+c$

where $\hat{\theta}$ represents the sample estimates of distribution parameters $\left(\mu_{\mathrm{L} \text {-CS }}, \mu_{\mathrm{L}-\mathrm{CK}}, \sigma_{\mathrm{L}-\mathrm{CS}}^{2}, \sigma_{\mathrm{L} \text {-CK }}^{2}\right.$, and $\left.\rho\right)$. Tables 3 and 4 , respectively, show the regression equations for the normal and Gumbel distributions, using both the probabilityweighted-moment estimator and the plotting-position estimator. For normal distribution using the probability- weighted-moment estimators, all regression coefficients for sample mean of $L$-skewness $\left(\hat{\mu}_{L \text {-cs }}\right)$ and correlation coefficient $r$ are nonsignificant. Therefore, for normal distribution, empirical relationships are needed for $\hat{\mu}_{L-C S}, \hat{\sigma}_{L-C S}^{2}$ and $\hat{\sigma}_{L-C K}^{2}$ only, if the probability-weighted-moment estimators are used, and both $\hat{\mu}_{L \text {-cs }}$ and $r$ are set to zero for different values of sample size $n$. For normal distribution using the plottingposition estimators and Gumbel distribution using both estimators (except correlation coefficient between $\tilde{\tau}_{3}$ and $\tilde{\tau}_{4}$ of the Gumbel distribution), sample estimates of all parameters are well correlated with the sample size. Therefore, $95 \%$ acceptance regions corresponding to any given sample size $n$ can be established using the empirical relationships in Tables 3 and 4 and Eq. (48). A simple example is given below to demonstrate usage of the established acceptance regions for GOF tests.

Suppose that a random sample of size $n=44$ is available, and the plotting-position sample $L$-skewness and $L$-kurtosis are calculated as $\left(\tilde{\tau}_{3}, \tilde{\tau}_{4}\right)=(0.214,0.116)$. We want to test whether the sample is originated from the Gumbel distribution. From the regression models for plotting-position estimators in Table 4, we find $\hat{\mu}_{L-C S}, \hat{\mu}_{L-C K}, \hat{\sigma}_{L-C S}^{2}, \hat{\sigma}_{L-C K}^{2}$, and $r$ to be, respectively, $0.1784,0.1369,0.005119,0.002924$, and 0.6039 . The Hotelling's $T^{2}$ is then calculated as 0.9908 using Eq. (45). Thus, the value of $T^{2}$ is much smaller than the threshold value $\chi_{2,0.05}^{2}=5.99$, and the null hypothesis that the random sample is originated from the Gumbel distribution is not rejected.

Table 3 Empirical relationships between the sample size and parameters of the bivariate distribution of sample $L$-skewness and $L$-kurtosis [normal distribution, $\tau_{3}=0, \tau_{4}=0.1226$ ]

\begin{tabular}{|c|c|c|c|c|c|c|c|c|}
\hline \multirow[t]{2}{*}{$\hat{\theta}$} & \multicolumn{4}{|c|}{ Probability-weighted-moment estimator $\left(t_{3}, t_{4}\right)$} & \multicolumn{4}{|c|}{ Plotting-position estimator $\left(\tilde{\tau}_{3}, \tilde{\tau}_{4}\right)$} \\
\hline & $\bar{a}$ & $b$ & c & $R^{2}$ & $a$ & $b$ & $c$ & $R^{2}$ \\
\hline$\hat{\mu}_{L-C S}$ & - & - & 0 & NA & 0.9042 & - & - & 0.9999 \\
\hline$\hat{\mu}_{L-C K}$ & 0.0313 & - & 0.1226 & 0.9193 & -0.6639 & - & 0.1226 & 0.9968 \\
\hline$\hat{\sigma}_{L-C S}^{2}$ & 0.1845 & 0.9221 & - & 1 & 0.1870 & - & - & 1 \\
\hline$\hat{\sigma}_{L-C K}^{2}$ & 0.0844 & 0.9959 & - & 1 & 0.0876 & -0.1500 & - & 0.9999 \\
\hline$r^{\operatorname{Len}}$ & - & - & 0 & NA & 2.2640 & - & - & 0.9938 \\
\hline
\end{tabular}

Regression model: $\hat{\theta}(n)=\frac{a}{n}+\frac{b}{n^{2}}+c$.

Cells marked by dash lines indicate corresponding coefficients are not included in the regression model. Also, bold numbers are designated values. For example, the regression model for $\hat{\mu}_{\mathrm{L}-\mathrm{CK}}$ of both estimators is $\hat{\mu}_{\mathrm{L}-\mathrm{CK}}(n)=\frac{a}{n}+0.1226$, and the correlation coefficient of $t_{3}$ and $t_{4}$ is assigned to be 0 .

Table 4 Empirical relationships between the sample size and parameters of the bivariate distribution of sample $L$-skewness and L-kurtosis [Gumbel distribution, $\tau_{3}=0.1699, \tau_{4}=0.1504$ ]

\begin{tabular}{|c|c|c|c|c|c|c|c|c|}
\hline \multirow[t]{2}{*}{$\hat{\theta}$} & \multicolumn{4}{|c|}{ Probability-weighted-moment estimator $\left(t_{3}, t_{4}\right)$} & \multicolumn{4}{|c|}{ Plotting-position estimator $\left(\tilde{\tau}_{3}, \tilde{\tau}_{4}\right)$} \\
\hline & $a$ & $b$ & c & $R^{2}$ & $a$ & $b$ & $c$ & $R^{2}$ \\
\hline$\hat{\mu}_{L-C S}$ & -0.1927 & - & 0.1699 & 0.9954 & 0.3539 & 0.7789 & 0.1699 & 0.9995 \\
\hline$\hat{\mu}_{L-C K}$ & -0.0426 & - & 0.1504 & 0.9469 & -0.5426 & -2.3331 & 0.1504 & 1.0 \\
\hline$\hat{\sigma}_{L-C S}^{2}$ & 0.2296 & 0.7454 & - & 1.0 & 0.2324 & -0.3159 & - & 1.0 \\
\hline$\hat{\sigma}_{L-C K}^{2}$ & 0.1295 & 1.0425 & - & 0.9999 & 0.1351 & -0.2840 & - & 1.0 \\
\hline$r$ & -1.5321 & - & 0.6063 & 0.9908 & - & - & 0.6039 & NA \\
\hline
\end{tabular}

Regression model: $\hat{\theta}(n)=\frac{a}{n}+\frac{b}{n^{2}}+c$.

Cells marked by dash lines indicate corresponding coefficients are not included in the regression model. Also, bold numbers are designated values. For example, the regression model for $\hat{\mu}_{\mathrm{L}-\mathrm{CK}}$ of $t_{4}$ is $\hat{\mu}_{\mathrm{L}-\mathrm{CK}}(n)=\frac{a}{n}+0.1504$, and the correlation coefficient of $\tilde{\tau}_{3}$ and $\tilde{\tau}_{4}$ is assigned to be 0.6039 . 
It is worthy to note that the rationale of choosing standard normal and standard Gumbel distributions for stochastic simulation in our study lies in the fact that certain parameters of the test statistics being considered are invariant to changes in the location and scale of the data from which they are constructed and thus are invariant to changes in the location and scale of the distributions which are used for simulations. However, the plotting-position $L$ moment estimators are biased and non-invariant estimators (Hosking and Wallis, 1997), and thus, the acceptance regions of the $L$-moments of standard normal or Gumbel distribution based on the plotting-position approach are not generally applicable for other nonstandard distributions. To circumvent such difficulties, the data used for GOF test should be preprocessed for normalization (zero mean and unit standard deviation), and then the normalized data be used for calculation of the plotting-position L-moment estimates.

\section{Validity check of the LMRD acceptance regions}

The sample-size-dependent acceptance regions established using empirical relationships described in the previous section are further checked for their validity. This is done by stochastically generating 10,000 random samples for both the standard normal and Gumbel distributions, with sample size $n=20,30,40,50,60,75,100,150,250,500$, and 1000 . For each set of 10,000 random samples, sample $L$-skewness and $L$-kurtosis pair, i.e. $\left(t_{3}, t_{4}\right)$ or $\left(\tilde{\tau}_{3}, \tilde{\tau}_{4}\right)$, were calculated and GOF-test was conducted using the corresponding sample-size-dependent $95 \%$ acceptance regions established using the empirical relationships and Eq. (48). The rejection rate, i.e. the type I error, is then calculated as

$\hat{\alpha}=N_{\mathrm{r}} / N$

where $N_{r}$ and $N$, respectively, represent the rejected and the total number of random samples.

For the sample-size-dependent 95\% acceptance regions to be valid, the rejection rate $\hat{\alpha}$ should be very close to

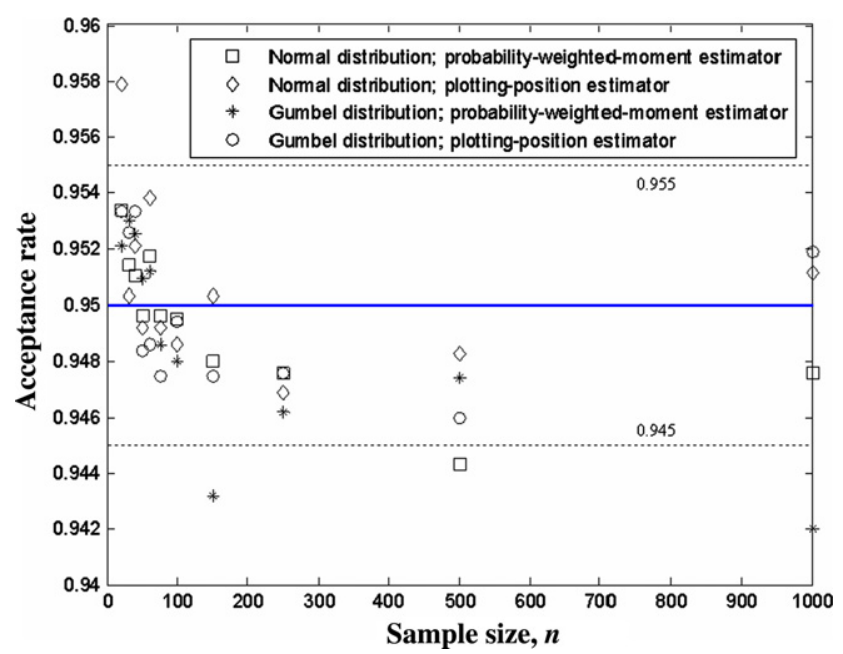

Figure 12 Acceptance rate of the validity check for samplesize-dependent $95 \%$ acceptance regions of sample $L$-skewness and $L$-kurtosis pairs. the level of significance $(\alpha=0.05)$ or the acceptance rate be very close to 0.95 . Results of the validity check are demonstrated in Fig. 12. For either type of distribution, the acceptance rates of both the probability-weighted-moment estimator and the plotting-position estimator, with respect to various sample sizes, nearly all fall within the very small range of $[0.945,0.955]$. Such results strongly indicate the validity of the sample-size-dependent $95 \%$ acceptance regions established using the empirical relationships in Tables 3 and 4 and Eq. (48), and that they can be applied to GOF test for random samples of any sample size between 20 and 1000 . It is also noteworthy that although results of the Mardia test suggested the bivariate normality assumption for sample $L$-skewness and $L$-kurtosis may not be adequate for smaller sample size $n$, their corresponding $95 \%$ LMRD acceptance regions still yield acceptance rates very close to 0.95 .

\section{Conclusions}

A detailed account of stochastic simulation for establishment of sample-size-dependent $95 \%$ acceptance regions of a GOF test based on L-moment-ratios is presented. Properties of the sample $L$-skewness and $L$-kurtosis estimated by the probability-weighted-moment estimator and the plotting-position estimator are discussed. The 95\% acceptance regions of the $L$-moments based GOF tests for the normal and the Gumbel distributions are further validated through a stochastic simulation process. A few concluding remarks are drawn as follows:

1. For moderate to large sample sizes (for example $n>60$ ), sample $L$-skewness and L-kurtosis, estimated by the probability-weighted-moment estimator or the plottingposition estimator, of the normal and the Gumbel distributions form a bivariate normal distribution.

2. For the normal and the Gumbel distributions, the asymptotic biases of the sample $L$-skewness and $L$-kurtosis of the probability-weighted-moment estimator and the plotting-position estimator are well correlated with the sample size, with the exception of the sample L-skewness of the normal distribution by the probabilityweighted-moment estimator which is considered to be unbiased.

3. Even though the probability-weighted-moment estimators of the sample $L$-skewness and $L$-kurtosis perform better in terms of the unbiasedness, the plotting-position estimators yield smaller variances for sample $L$-skewness and $L$-kurtosis of both distributions.

4. The sample-size-dependent $95 \%$ acceptance regions established in this study have been validated through a stochastic simulation process and can be applied to GOF test for random samples of any sample size between 20 and 1000 .

\section{Acknowledgements}

We gratefully acknowledge the National Science Council of Taiwan, R.O.C. for financially supporting this research. 
The corresponding author is also grateful for the Kyoto University, Japan for extending him a 6-month guest professorship during which this manuscript was prepared. We also thank three anonymous reviewers for their insightful comments and suggestions which are very helpful in preparing the final form of this paper.

\section{References}

Andrews, D.F., Gnanadesikan, R., Warner, J.L., 1973. Methods of assessing multivariate normality. In: Krishnaiah, P.R. (Ed.), Multivariate Analysis. Academic Press, New York.

Bowman, K.O., Shenton, L.R., 1975. Omnibus test contours for departures from normality based on $\sqrt{b_{1}}$ and $b_{2}$. Biometrika 62 , 243-250.

Bowman, K.O., Shenton, L.R., 1986. Moment $\left(\sqrt{b_{1}}, b_{2}\right)$ techniques. In: D’Agostino, R.B., Stephens, M.A. (Eds.), Goodness-of-Fit Techniques. Marcel Dekker, New York.

Chow, V.T., 1951. A general formula for hydrologic frequency analysis. Transaction of American Geophysics Union 32, $231-$ 237.

Chow, K.C., Watt, W.E., 1994. Practical use of the L-moments. In: Hipel, K.W. (Ed.), Stochastic and Statistical Methods in Hydrology and Environment Engineering. Kluwer Academic Publishers., Boston, MA, USA.

Cox, D.R., 1968. Notes on some aspects of regression analysis (with discussion). Journal of the Royal Statistical Society Series A 131, 265-279.

Cox, D.R., Small, N.J.H., 1978. Testing multivariate normality. Biometrika 65 (2), 263-272.

D’Agostino, R.B., Pearson, E.S., 1973. Tests for departure from normality. Empirical results for the distributions of $b_{2}$ and $\sqrt{b_{1}}$.. Biometrika 60, 613-622.

D’Agostino, R.B., Stephens, M.A., 1986. Goodness-of-Fit Techniques. Marcel Dekker, New York.

Fisher, R., 1930. The moments of the distribution for normal samples of measures of departures from normality. Proceedings of the Royal Society Series A 130, 16-28.

Greenwood, J.A., Landwehr, J.M., Matalas, N.C., Wallis, J.R., 1979. Probability weighted moments: definition and relation to parameters of several distributions expressible in inverse form. Water Resources Research 15, 1049-1054.
Haan, C.T., 2002. Statistical Methods in Hydrology. lowa State Press, Ames, lowa.

Heiberger, R.M., Holland, B., 2004. Statistical Analysis and Data Display: An Intermediate Course with Examples in S-Plus, R, and SAS. Springer Science+Business Media, New York, USA.

Hosking, J.R.M., 1990. L-moments: analysis and estimation of distributions using linear combinations of order statistics. Journal of the Royal Statistical Society Series B 52 (1), 105-124.

Hosking, J.R.M., Wallis, J.R., 1993. Some statistics useful in regional frequency analysis. Water Resources Research 29 (2), 271-281.

Hosking, J.R.M., Wallis, J.R., 1995. A comparison of unbiased and plotting-position estimators of L-moments. Water Resources Research 31 (8), 2019-2025.

Hosking, J.R.M., Wallis, J.R., 1997. Regional Frequency Analysis: An Approach based on L-moments. Cambridge University Press, Cambridge, UK.

Kite, G.W., 1988. Frequency and Risk Analysis in Hydrology. Water Resources Publications.

Kottegoda, N.T., 1980. Stochastic Water Resources Technology. Macmillan, London, UK.

Mardia, K.V., 1970. Measures of multivariate skewness and kurtosis with applications. Biometrika 57 (3), 519-530.

Mardia, K.V., Kent, J.T., Bibby, J.M., 1979. Multivariate Analysis. Academic Press, New York.

Vogel, R.M., Fennesset, N.M., 1993. L-moments diagrams should replace product moment diagrams. Water Resources Research 29 (6), 1745-1752.

Vogel, R.M., McMahon, T.A., Chiew, H.S., 1993. Flood flow frequency model selection in Australia. Journal of Hydrology 146, 421-449.

Vogel, R.M., Wilson, I., 1996. Probability distribution of annual maximum, mean, and minimum streamflows in the United States. Journal of Hydrologic Engineering 1 (2), 69-76.

Wallis, J.R., 1988. Catastrophes, computing, and containment: living with our restless habitat. Speculation in Science and Technology 11 (4), 295-323.

Wu, Y.C., 2005. Establishing acceptance regions for goodness-of-fit test by stochastic simulation. Master Thesis. Department of Bioenvironmental Systems Engineering, National Taiwan University (in Chinese). 